\title{
The Role of Microbiome in Sexually Transmitted Infections
}

\author{
Felix Hartanto ${ }^{1}$, Afif Nurul Hidayati ${ }^{1,2 *}$ \\ afif_nurulhidayati@yahoo.com \\ ${ }^{1}$ Department of Dermatology and Venereology, Faculty of Medicine, Universitas Airlangga, Dr. Soetomo General Hospital, Jl. \\ Prof Dr. Moestopo No. 6-8, Surabaya, 60286, Indonesia \\ ${ }^{2}$ Universitas Airlangga Hospital, Jl. Dharmahusada Permai, Surabaya, 60115, Indonesia
}

\begin{abstract}
Background: Several studies have shown that abnormal vaginal flora associated with sexually transmitted infections (STIs).

Content: Microbiome is an ecological ecosystem of symbiotic, commensal, and pathogenic organisms that live in human body space. According to research, uncircumcised males are more likely to be colonized by gram-positive and gram-negative bacteria or other diseases. The vaginal community is divided into five microbiomes: community-state type (CST) I is dominated by L. crispatus; CST II by L. gasseri; CST III by L. iners; and CST V by L. jensenii. CST IV, on the other hand, is diverse and has a greater number of obligate anaerobic bacteria. Lactobacillus generates bacteriocin and lactic acid, which help to inhibit bacterial development by keeping vaginal $\mathrm{pH}$ low ( $\mathrm{pH} 3.0$ - 4.5). Bacterial vaginosis (BV) is a syndrome that occurs due to dysbiosis in vaginal microbiome. The protective mechanism by Lactobacillus compromises in BV, which becomes an entry point for pathogenic organisms. Protection against $\mathrm{C}$. trachomatis infection is mainly generated by lactic acid produced by Lactobacillus. The low $\mathrm{pH}$ is also known to inhibit the survival of $\mathrm{N}$. gonorrhoeae. Protection mechanism by Lactobacillus against viral infections such as HIV, HSV-2, and HPV can occur direct or indirectby several complex and integrated mechanisms.
\end{abstract}

Conclusion: microbiome-based approaches have promising outcomes for both preventive and curative measures for sexually transmitted infections.

Keywords : microbiome, Lactobacillus, sexually transmitted infection

\section{BACKGROUND}

Sexually transmitted infections (STIs) are reported by the World Health Organization (WHO) nearly 1 million cases worldwide each day and about 376 million new cases of infection with 1 in 4 STIs, namely chlamydia, gonorrhoea, syphilis, and trichomoniasis each year. ${ }^{[1]}$

Studies regarding protective mechanisms generated by a healthy vaginal ecosystem against sexually transmitted infections show inconsistent results. Main factors influencing the results are lower amount of lactic acid-producing bacteria (Lactobacillus), local cytokine production, and high vaginal $\mathrm{pH}$. Several studies have shown that abnormal vaginal flora is associated with gonorrhoea, chlamydia, and trichomoniasis invasion. Furthermore, the makeup of the vaginal microbiome has been linked to several sexually transmitted viral diseases (including HIV, Human papillomavirus, Herpes Simplex Virus, and Cytomegalovirus). ${ }^{[2-4]}$

Regarding many roles of the microbiome in human genitalia as mentioned above, it is important to create a literature review aiming discussions of theories and scientific evidence that can be used as a basis for preventive and curative efforts against several sexually transmitted infections which will be discussed in this review.

\section{CONTENT}

In 1988, Whipps et al. described 'microbiome' as a combination of the words 'micro' and 'biome', naming 'characteristics of microbial communities' in the 'habitats of different physiochemical properties'. The definition currently most cited is by Lederberg, who describes the microbiome in an ecological context, as a community of commensal, symbiotic and pathogenic microorganisms in human body space or other environments. Marchesi and Ravel focused on defining the microbiome at the genome level and the expression patterns of microbial and 
viral genes as well as proteomes in certain environments and the prevailing biotic and abiotic conditions. A combination of ecological, host, and genomic definitions describe microbiome as an ecological community of symbiotic, commensal, and pathogenic microorganisms that physically share the living space in the human body. ${ }^{[5]}$

Sexually Transmitted Infections (STIs) are infections spread from one person to another via sexual contact. Contact usually occurs through vaginal, oral, and anal sex or other intimate physical contacts since some STIs, such as herpes and Human Papillomavirus (HPV), are transmitted through skin-to-skin contact. ${ }^{[6-7]}$ In general, STIs are classified based on aetiology, which are bacteria, virus, and other organisms. This paper will discuss more about sexually transmitted infections that can be affected by microbiota imbalance in the genital organs of both men and women.

\section{Microbiome in Male Genitalia}

According to Mandar et al., the male genital microbiota is primarily located in the lower genital tract. Bowie et al. ${ }^{[8]}$ studied 69 Caucasian males with Nongonococcal Urethritis (NGU) and 39 controls, obtaining aerobic and anaerobic cultures from each subject's urethra. The most frequent bacteria include Staphylococcus epidermidis, Corynebacterium spp., Lactobacillus, anaerobic Gram-positive cocci, and Bacteroides spp. ${ }^{[8]}$

Willén et al. collected specimens from six sites in 97 healthy men scheduled for vasectomy and discovered that $71 \%$ of the strains colonizing the coronal sulcus were also prevalent in the urethra, implying that the distal urethra is occupied by a bacterial flora similar to that found in the coronal sulcus. The most common bacteria groupings are coagulase-negative staphylococci and streptococci. ${ }^{[8]}$

Schneider et al. conducted aerobic and anaerobic culturing on coronal sulcus and urethral groove samples from circumcised and uncircumcised males $(\mathrm{n}=315)$ in South India. Nearly half $(48 \%)$ of the patients were HIVpositive, $36 \%$ were tuberculosis-positive, and $16 \%$ were uninfected. Gram-negative bacteria were more prevalent in HIV- and tuberculosis-infected males than in controls. Uncircumcised males are more likely to be infected with gram-positive bacteria (Enterococcus spp., Staphylococcus aureus), gram-negative bacteria (Pseudomonas aeruginosa, Klebsiella spp., Escherichia coli), or other diseases (Candida albicans, Clostridium spp.). ${ }^{[8]}$

\section{Microbiome in Female Genitalia}

The ecology of the vagina depends on the interaction of the vaginal environment and relatively limited flora species, especially Lactobacillus spp. Studies reported that vaginal community grouped into five main vaginal microbiomes, defined as community-state type (CST). Lactobacillus crispatus in CST I, Lactobacillus gasseri in CST II, Lactobacillus iners in CST III, and Lactobacillus jensenii in CST V dominated each of these types of community states. Whereas, CST IV bacteria were varied and included a greater number of obligate bacilli such as Gardnerella, Atopobium, Prevotella spp., and others. The CST IV is divided into IV-A and IV-B subtypes. Both have a heterogeneous composition, but CST IV-B contains less Lactobacillus and more anaerobic bacilli related to bacterial vaginosis. ${ }^{[9]}$

\begin{tabular}{|c|c|c|c|c|c|c|c|c|c|}
\hline \multicolumn{2}{|c|}{ Type I } & \multicolumn{2}{|c|}{ Type II } & \multicolumn{2}{|c|}{ Type III } & \multicolumn{2}{|c|}{ Type IV } & \multicolumn{2}{|c|}{ Type V } \\
\hline Species & Specificity & Species & Specificity & Species & Specificity & Species & Specificity & Species & Specificity \\
\hline L crispatus & 0.952 & L. gasseri & 0.923 & Liners & 0.778 & Prevotella & 0.783 & L jensenii & 0.915 \\
\hline Lactobacillales_6 & 0.944 & Lactobacillales_1 & 0.710 & Lactobacillales_2 & 0.724 & Dialister & 0.750 & Lactobacillates_5 & 0.456 \\
\hline Clostridium & 0.114 & Lmaginalis & 0.369 & Lactobacillales_5 & 0.293 & Atopobium & 0.678 & Lactobacillales_7 & 0.243 \\
\hline Lactobacillus_2 & 0.099 & Anaeracaccis & 0.244 & Finegoldin & 0.036 & Eggerthella & 0.664 & Propionibacterium & 0.203 \\
\hline Staphylococcus & 0.083 & Peptoniphilus & 0.214 & Staphylococcas & 0.035 & Sneathia & 0.662 & Streptococcus & 0.122 \\
\hline Lvaginalis & 0.077 & Lactobacillus_3 & 0.155 & Ureaplasma & 0.034 & Parvimonas & 0.659 & Enhydrobacter & 0.087 \\
\hline Lactobuaillales_5 & 0.070 & Gardnerella & 0.153 & Conyebucterium & 0.032 & Ruminocaccacene_3 & 0.655 & Corynebacterium & 0.070 \\
\hline Liners & 0.060 & Finegoldia & 0.149 & Aerocoucus & 0.031 & Megasphiaera & 0.655 & Acinetobacter & 0.056 \\
\hline Lactobacillales_2 & 0.033 & Bifidobacterium & 0.144 & Lactobacillales_? & 0.026 & Prevotellacane_2 & 0.601 & Fingogoldia & 0.050 \\
\hline Exiguolacterium & 0.031 & Uraplasma & 0.114 & Lactobacillus_2 & 0.025 & Mobiluncus & 0.504 & Skermanella & 0.048 \\
\hline Mean (M) & 0.246 & & 0.318 & & 0.201 & & 0.661 & & 0.225 \\
\hline Variance (V) & 0.137 & & 0.077 & & 0.091 & & 0.006 & & 0.075 \\
\hline $\mathrm{SAI}=\mathrm{V} / \mathrm{M}$ & 0.558 & & 0.242 & & 0.451 & & 0.009 & & 0.333 \\
\hline SAI ratio to type IV & 62 & & 27 & & so & & 1 & & 37 \\
\hline
\end{tabular}

Fig. 1 The community-state type in the female genitalia. ${ }^{[10]}$ 
Other research indicates that a microbiome dominated by Lactobacillus species other than L. iners is beneficial to vaginal health. The presence of vaginal Lactobacillus, particularly L. crispatus, is significantly associated with the lack of bacterial vaginosis. Lactobacillus is also beneficial to vaginal health since it produces lactic acid, $\mathrm{H}_{2} \mathrm{O}_{2}$, disinfectants, antibacterial compounds, and bacteriocin. Bacteriocin can kill urogenital infections in vitro under various circumstances, and lactic acid, in addition to maintaining a very acidic $\mathrm{pH}$, can function as an antibacterial agent by breaking bacterial cell membranes and activating human defense against bacterial lipopolysaccharide. ${ }^{[9]}$

\section{Microbiome and Immune System}

Cervicovaginal fluid (CVF) keeps the vaginal mucosal environment maintained by including epithelial cell products like mucin as well as antimicrobial compounds like Elafine, B-Defensin, Lipocalin, Secretory Leukocyte Protease Inhibitor (SLPI), and IgA and IgG antibodies. CVF continually lubricates the epithelium and serves as the initial defence against pathogen colonization via mucin activity, which traps microorganisms and bind them to antibodies. ${ }^{[11]}$

The vaginal microbiota is distinct, dominated mostly by Lactobacillus species that generate lactic acid and bacteriocin, which contribute to the prevention of bacterial development and the maintenance of a low vaginal $\mathrm{pH}$ (pH 3.0 - 4.5). Furthermore, Lactobacillus species sticking to epithelial surfaces inhibit pathogens from adhering and infecting epithelial cells, promote phagocytosis, facilitate clearance, and control inflammatory processes. ${ }^{[11]}$

In addition to epithelial cells and bacteria, the vaginal ecosystem includes innate and adaptive immune cells such as macrophages, neutrophils, langerhans cells, dendritic cells, natural killer (NK) cells, and T and B lymphocytes. The most frequent antigen presenting cells (APCs) in the vaginal environment are dendritic cells and monocytes. Inflammation and immune cell activation are rigorously governed by Pattern Recognition Receptor (PRR) expression and controlled by endocrine signals throughout the reproductive tract. ${ }^{[1]}$

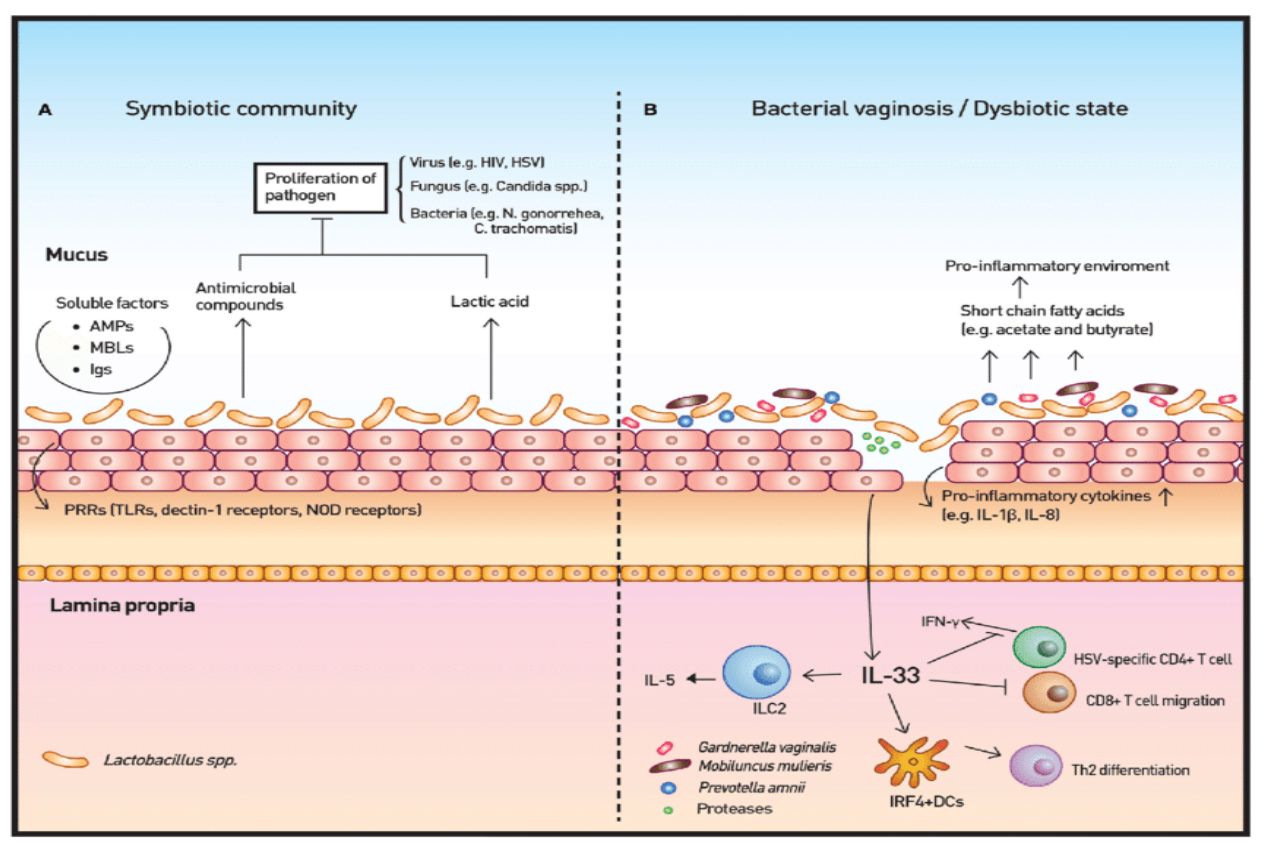

Fig. 2 Relationship of vaginal microbiota and immunity. ${ }^{[12]}$

When Lactobacillus is absent and microbial diversity increases, changes in immune and epithelial homeostasis occur, inducing various defence mechanisms such as: (a) immune cell recruitment; (b) production of proinflammatory cytokines and chemokines; and (c) decreased CVF viscosity due to the production of mucindegrading enzymes. ${ }^{[11]}$ 


\section{Role of the Microbiome in Defence Against Sexually transmitted infection}

Until recently, CVF coated with Lactobacillus is still considered as the optimal environment, but recent molecular studies show that not all Lactobacillus has similar features. L. crispatus is associated with the antiinflammatory profile of CVF and protection from developing anaerobic dysbiosis. In contrast, L. iners does not appear to protect from anaerobic dysbiosis and often coexist with BV-related anaerobes. ${ }^{[13]}$

Epidemiological studies suggest that BV, vulvovaginal candidiasis (CVV), and other STIs are typically bidirectionally linked. Behavioral and biological variables can elucidate this connection. For starters, several of these problems have risk factors for sexual transmission. While BV and CVV are frequently regarded as non-STIs because they can arise in the absence of sexual activity, it is now evident that route of transmission of the organisms involved does have a role, particularly in sexual encounters with uncircumcised male partners. Second, the majority of dysbiosis and CVV induce mucosal barrier abnormalities, which reduce mucus and vaginal secretions' capacity to capture or deactivate microorganisms. STI and anaerobic dysbiosis commonly coincide, although CVV appears to develop more frequently in the presence of Lactobacillus predominance than in the absence of anaerobic dysbiosis, as illustrated in Figure 3. ${ }^{[13]}$

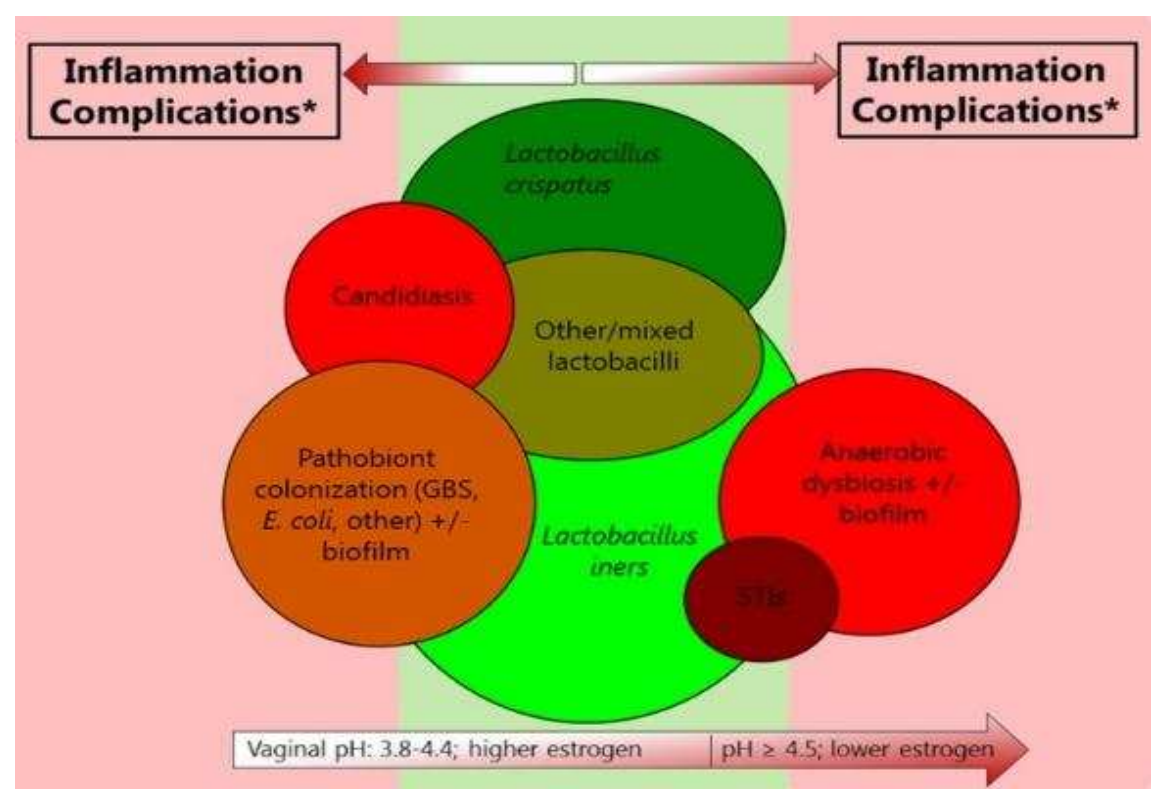

Fig. 3 Visualization of a two-way relationship between various urogenital conditions

\section{Microbiome and Bacterial Vaginosis}

Amsel et al. established BV diagnostic criteria, and their clinical applicability has lately expanded. At least three of the following four properties are required: homogenous vaginal discharge, $\mathrm{pH} 4.5$, fishy odor of volatile amines (evaluated with $\mathrm{KOH}$ solution), and presence of clue cells (coated squamous epithelial cells with bacteria) on microscopic examination. ${ }^{[14]}$

A number of putative bacterial species have been discovered in the past utilizing culture-based and molecular techniques. Each species has distinct features that must be taken into account when assessing the vaginal microbiome and the continuous interactions. Figure 4 depicts a schematic connection between these three variables (host genome expression, vaginal microbiota, and vaginal/systemic exposure). 


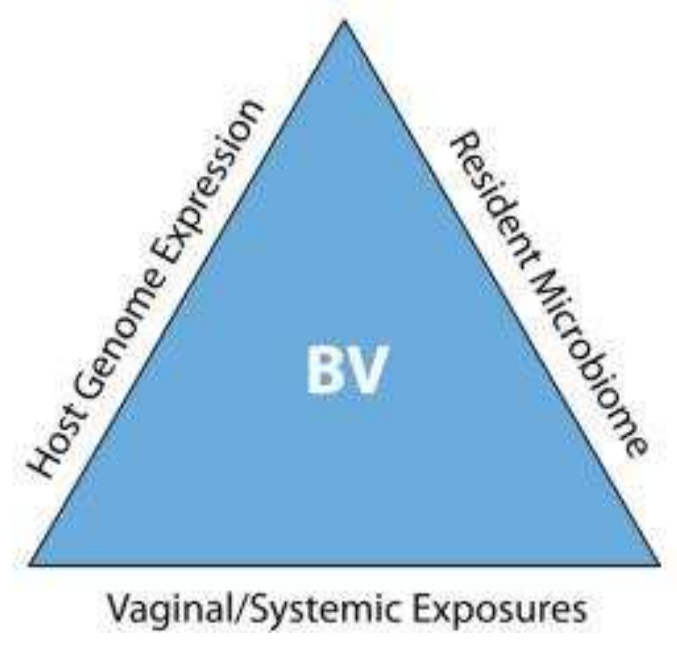

Fig 4. Schematic picture of bacterial vaginosis (BV) syndrome.

A study by Widiatma et al. in 2021 in 30 BV patients diagnosed in the dermatology outpatient clinic of Dr. Soetomo Hospital, shows that all samples of patients with BV experienced shifting in the vaginal microbiome from Lactobacillus to Gardnerella spp. and other anaerobic bacteria. However, it also stated that giving additional probiotic supplementation in the form of L. plantarum for one month on standard Metronidazole therapy did not give significantly different results on patients' recovery compared to the control or placebo group. ${ }^{[15]}$

\section{a. Gardnerella vaginalis}

Through traditional culture techniques and molecular-based investigations, a number of studies have established the presence of G. vaginalis in women with or without BV. Overall G. vaginalis DNA quantity, on the other hand, was linked to three of Amsel's four criteria, including amine odor, elevated $\mathrm{pH}$, and the presence of clue cells. The biofilm-forming type of G. vaginalis was shown to be highly linked between sexual partners, implying the possibility of sexual transmission. ${ }^{[14]}$

Many research have emphasized on probable virulence factors that might explain G. vaginalis pathogenic potential and probable participation in BV. G. vaginalis virulence factors are similar to adhesin, which is generated by Mycoplasma and is involved in adhesion to human tissue, and cytolysin, which induces apoptosis in human epithelial cells by the activation of protein kinase pathway. Furthermore, G. vaginalis generates sialidase, prolidase, and putressin, that might reduce mucosal protective components like mucin and lead to vaginal epithelial cell exfoliation. ${ }^{[14]}$

\section{b. Atopobium vaginae}

A. vaginae is found in 96 percent of women with BV but only in 12 to 19 percent of women who do not have $\mathrm{BV}$ and Amsel's four clinical criteria were all linked to A. vaginae. The composition of the biofilm adhering to the vaginal mucosa in $\mathrm{BV}$ individuals revealed that $\mathrm{A}$ vaginale was present in $70 \%$ of the subjects. The connection of A. vaginale with biofilm development and metronidazole resistance might clarify why BV treatment fails. ${ }^{[14]}$

\section{c. Prevotella and Porphyromonas}

Prevotella and Porphyromonas are classified as gram-negative and immotile bacteria. A number of investigations using Gram staining, culture, and molecular methods have found these bacteria in almost all women with and without BV.

Prevotella is also correlated to a positive whiff test, which is one of the Amsel's criteria. Positive whiff test resulted from the synthesis of polyamines such as trimethylamine, cadaverine, and putrescine, which raise the $\mathrm{pH}$ of the vaginal fluid. Prevotella also generates fibrinolysin and collagenase, that can breakdown the mucosal layer, as well as prolidase and sialidase, which cause vaginal shedding. ${ }^{[14]}$ 


\section{d. Sneathia dan Leptotrichia}

Sneathia has been found in vaginal samples from both BV and non-BV women. S. amnii was discovered in $40 \%$ of 736 women who participated in the Human Microbiome Project. S. amnii and S. sanguinegens were also discovered to co-occur frequently in the same population. ${ }^{[14]}$

Sneathia and Leptotrichia are significant in BV. Srinivasan et al. discovered that S. amnii is favorably connected to all four Amsel's criteria. The fact of Sneathia produces collagenase and fibrinolysin, which compromises the mucosal barrier and facilitates the discharge of epithelial cells in vagina, may explain its connection with the occurrence of clue cells. ${ }^{[14]}$

\section{e. Mobiluncus}

M. mulieris is the most common organism found in healthy women, but M. curtisii was found in $65.3 \%$ of $\mathrm{BV}$ infections. The prevalence and persistence of $\mathrm{M}$. curtisii are also linked to treatment failure.

\section{f. Mycoplasma and Ureaplasma}

M. genitalium causes symptomatic urethritis and cervicitis, whereas M. hominis causes BV. M. hominis is present in modest quantities in a healthy vagina, but its concentrations surged 10,000 fold in BV women. ${ }^{[14]}$

\section{Microbiome and Chlamydia trachomatis Infection}

The special feature found in Lactobacillus spp. is production of $\mathrm{H}_{2} \mathrm{O}_{2}$ and lactic acid which have antimicrobial properties and bacterial growth inhibition as illustrated in Figure 5. Lactic acid has showed as main inhibitor of C. trachomatis, whereas $\mathrm{H}_{2} \mathrm{O}_{2}$ inhibits BV-related bacteria such as M. genitalium. Gong et al. in 2014 hypothesized three possible mechanisms responsible for the effects of lactic acid on Chlamydia: (a) damage to surface molecules; (b) membrane damage; and (c) internal metabolic disorders. ${ }^{[16]}$

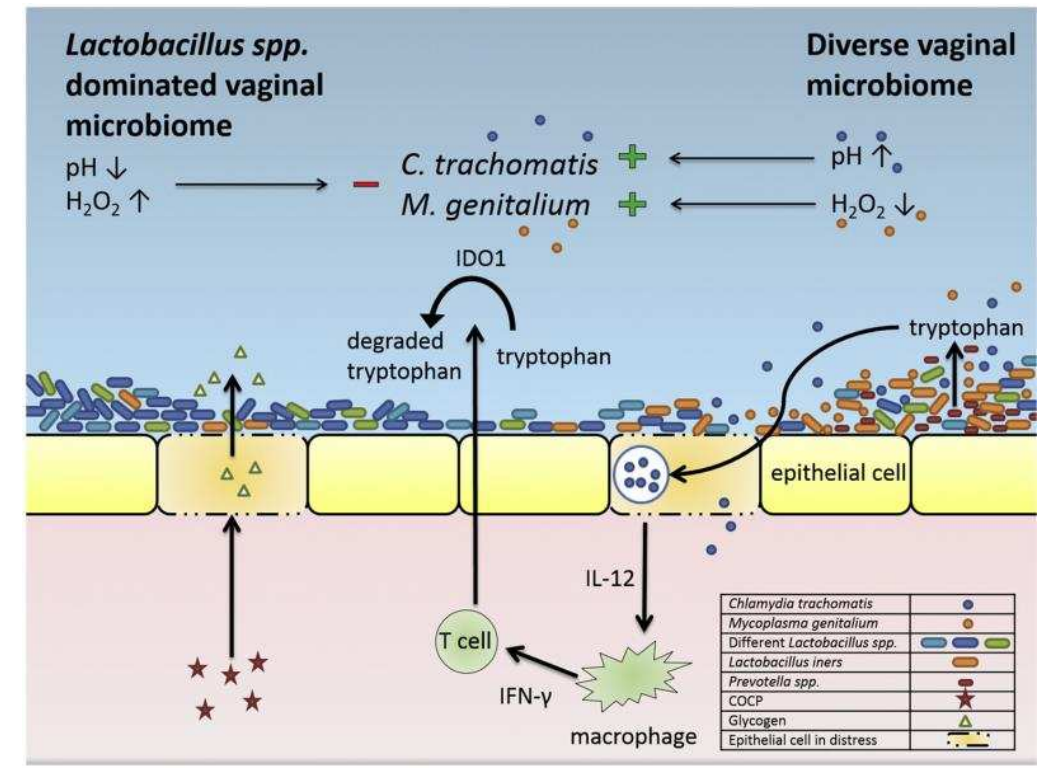

Fig. 5 Protection of the vaginal microbiome against C. trachomatis and M. genitalium.

However, not all Lactobacillus spp. is competent in warding off C. trachomatis. The research initiated by Van Houdt et al. found that vaginal microbiome dominated by $\mathrm{L}$. iners has low protection against $\mathrm{C}$. trachomatis infection because $\mathrm{L}$. iners produced less $\mathrm{H}_{2} \mathrm{O}_{2}$ and lactic acid compared to other Lactobacillus spp. ${ }^{[16]}$ 


\section{Microbiome and Neisseria gonorrhoeae Infection}

N. gonorrhoeae is an oxidase-positive gram-negative cocci bacteria that causes gonorrhoea, which is one of the most prevalent bacterial STIs globally. In most women, N. gonorrhoeae infection is often asymptomatic, and if it remains unknown and untreated, it will provide a significant reservoir for further transmission. ${ }^{[17]}$

It is known that in $\mathrm{pH}$ 6.4-7.3 the gonococcal bacteria can grow well, while at lower $\mathrm{pH}(4.8-5.0)$ there is a significant decrease in gonococcal survival. In addition, it has been reported that inhibition of N. gonorrhoeae by vaginal Lactobacillus due to lactic acid production as well as the modulation of bioactive substances in an acidic environment. At $\mathrm{pH}<4.0$, there is complete elimination of the viability of $\mathrm{N}$. gonorrhoeae, even only a short contact time (as shown in Table 1). ${ }^{[17]}$

Table 1. Correlation between $\mathrm{pH}$ value and the effect of Lactobacillus spp. on N. gonorrhoea $\mathrm{e}^{[17]}$

\begin{tabular}{llll}
\hline $\mathbf{p H}$ & Lactobacillus strain & $\begin{array}{l}\text { Anti-gonococcal effect at } \\
\text { th }\end{array}$ & $\begin{array}{l}\text { Anti-gonococcal effect at } \\
\mathbf{6 0}^{\text {th }} \text { minute }\end{array}$ \\
\hline$>4,5$ & L. gasseri BC10 & $5 \%$ gonococcal viability & $70-90 \%$ gonococcal \\
& L. vaginalis BC16 & reduction & viability reduction \\
$4-4,5$ & L. crispatus BC3 & $70-99 \%$ gonococcal & $100 \%$ gonococcal viability \\
& L. gasseri BC9, BC12, BC14 & viability reduction & reduction \\
& L. vaginalis BC17 & & \\
$<4$ & L. crispatus BC1, BC4-BC8 & $100 \%$ gonococcal viability & $100 \%$ gonococcal viability \\
& L. gasseri BC13 & reduction & reduction \\
\hline
\end{tabular}

Lactobacillus spp. in the vagina acts as a defence against $\mathrm{N}$. gonorrhoeae through a various mechanisms: (a) production of various antimicrobial compounds secreted by vaginal fluids; (b) aggregation ability of Lactobacillus spp.; (c) release of free component derivatives from Lactobacillus spp. (e.g. released surface components [RSC] which have biosurfactant activity). During interactions with N. gonorrhoeae, Surface components of biosurfactants, especially those from high aggregation capabilities Lactobacillus, can disrupt gonococci and reduce their viability. In contrast, non-aggregating Lactobacillus strains are completely ineffective against gonococci, despite the presence of RSC biosurfactant activity. ${ }^{[17]}$

\section{Microbiome and Human Immunodeficiency Virus Infection}

The Lactobacillus-dominated vaginal microbiota is important in the protection of a variety of sexually transmitted diseases, including the Human Immunodeficiency Virus (HIV). The vaginal microbiota has a direct inhibitory effect on HIV through the formation of lactic acid, $\mathrm{H}_{2} \mathrm{O}_{2}$, bacteriocin, and lectin molecules. In addition, as indicated in Figure 6, indirect processes such as the inhibition of bacteria linked with BV development, immune system activation, and defense activity by epithelial cells occur. ${ }^{[18]}$ 


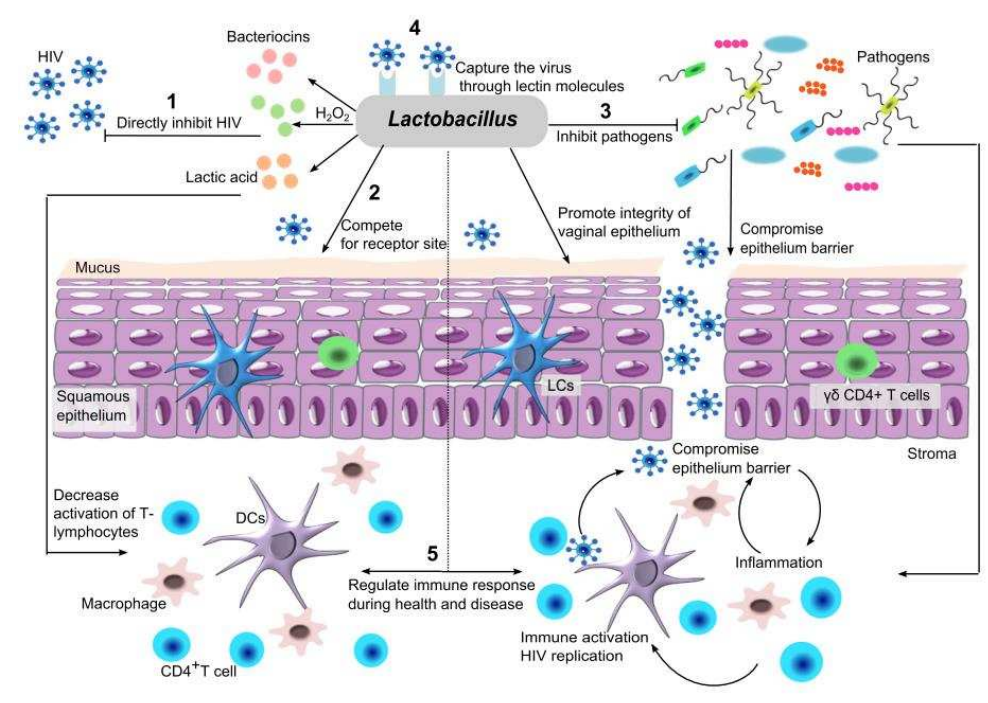

Fig. 6 Hypothesis of the mechanisms of vaginal Lactobacillus to prevent HIV infection in human. ${ }^{[18]}$

G. vaginalis generates numerous cytolysin classes, including vaginolysins, which can stimulate the protein kinase pathway in vaginal epithelial cells, resulting in cell death. Gardnerella spp. also often produces prolidase, putrescine, and sialidase, which reduce mucin, allowing for better microbial adhesion and biofilm development, as well as contributing to epithelial cell exfoliation. P. bivia, on the other hand, produces prolidase, sialidase, fibrinolysin, and collagenase. ${ }^{[19-20]}$

A metagenomic study of $16 \mathrm{~S}$ sequences from females with BV reveals that $\mathrm{P}$. bivia lipopolysaccharide is the best indicator of vaginal inflammation and HIV risk in females. Lipopolysaccharide binds to TLR-4 and CD14 to activate the NF-kB cytokine pathway. As a result, it is conceivable that these anaerobic bacteria play a significant role in vaginal inflammation, which may lead to poor defense and raise the risk of HIV in women with BV. ${ }^{[19-20]}$

\section{Microbiome and Herpes Simplex Virus-2 Infection}

Herpes Simplex Virus-2 (HSV-2) infection is a significant cofactor in HIV transmission and, similar to HIV, this infection is associated with changes in the microbiome in the vagina. A 2003 British study involving 520 women with a history of BV shows significant results linking vaginal microbiome dysbiosis with HSV-2 seropositivity. According to a cohort study, the increasing frequency of HSV-2 and BV infection is correlated. The relationship between BV and HSV-2 infection can be bidirectional, and BV is accountable for an increase in the incidence of HSV-2 reactivation in the female vaginal tract. ${ }^{[21]}$

A healthy vaginal microbiota can defend against viral infection through direct impacts or through natural defence components present in the vaginal ecosystem. Lactobacillus-produced hydrogen peroxide $\left(\mathrm{H}_{2} \mathrm{O}_{2}\right)$ acts as a natural microbicide in the vaginal environment and is harmful to a variety of species, including HIV and HSV2. Lactic acid, which is generated by all Lactobacillus species, has the ability to permanently inactivate HSV $-2 .{ }^{[21]}$ The gel coating that naturally covers the vaginal and cervical epithelium protects women against viral infections by its ability in capturing HSV virus. While in BV, it is known that many of the bacteria associated with BV generate increased amounts of sialidase, mucinase, and other mucin-degrading enzymes, that can compromise those natural abilities. ${ }^{[21]}$

\section{Microbiome and Human Papillomavirus Infection}

Several cross-sectional studies indicate that L. crispatus is more prevalent in females without HPV infection and cancer lesions, but L. iners and non-Lactobacillus species are more common in HPV-infected females and cancer patients. A recent meta-analysis also shown that a vaginal microbiome dominated by non-Lactobacillus or L.iners species is more associated with HPV infection and dysplasia than L. crispatus. ${ }^{[22]}$ 


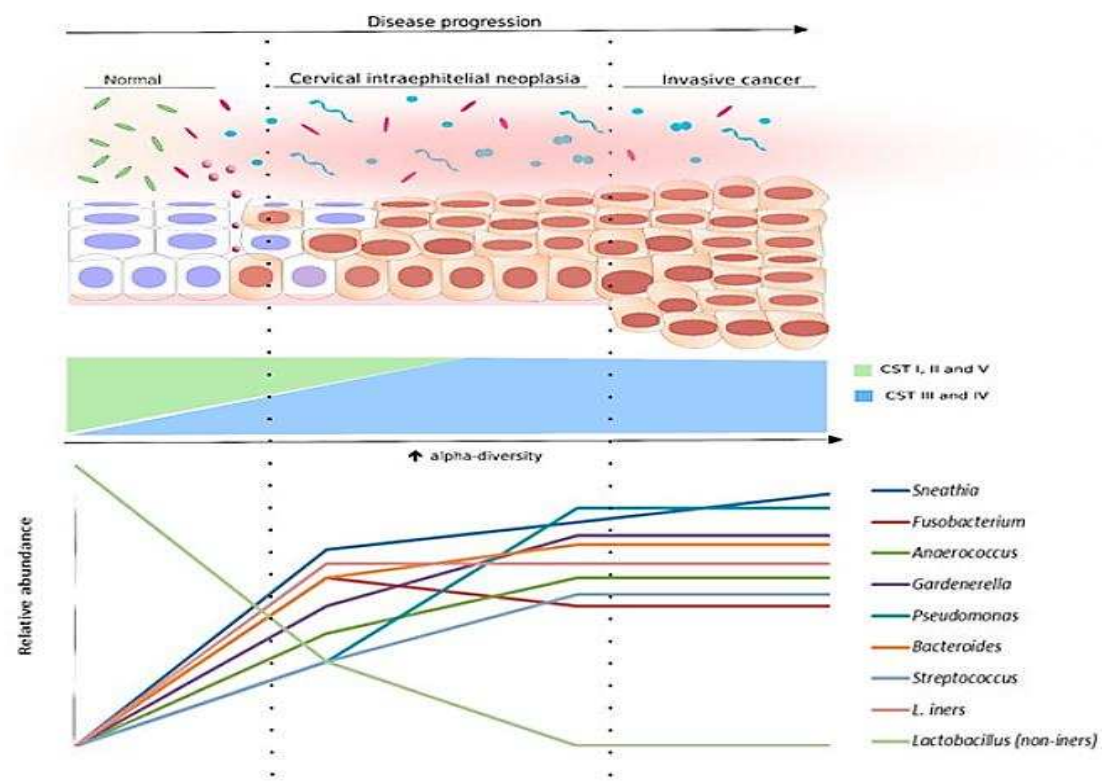

Fig. 7 Distribution of bacterial diversity in the development of intraepithelial neoplasia. ${ }^{23}$

Studies have revealed several methods through which BV can cause HPV infection to persist. A decrease in the quantity of lactic acid generating Lactobacillus results in an abnormally high $\mathrm{pH}(>4.5)$, which promotes bacterial overgrowth and a decrease in the protective flora. When this happened, the immune system's ability to fight viral infection is compromised. $\mathrm{BV}$ is also linked to an increase in the production of epithelial layer degrading enzymes, which allow HPV infection. Women with BV had higher amounts of the cytokine interleukin (IL)-1 and lower levels of the anti-inflammatory molecule SLPI (secretory leukocyte protease inhibitor). All of these BVrelated bacterial, mucosal, and immunological problems enhance susceptibility to HPV infection and the development of high-grade intraepithelial cancers / lesions. ${ }^{[23]}$

\section{Management of the Genitalia Microbiome as a Prevention Against Sexually Transmitted Infections}

Preventing STIs requires BV therapy, even in asymptomatic patients. Unfortunately, the CDC-recommended $\mathrm{BV}$ treatment, including metronidazole $500 \mathrm{mg}$ orally twice a day for 7 days or metronidazole / clindamycin gel / vaginal cream intravaginally at night for 7 days, does not appear to be helpful for limiting $\mathrm{BV}$ recurrence in the majority of patients. Those who underwent the CDC's regimen had a significant risk of recurrence after 6-12 months. Because the vaginal Lactobacillus population is seldom rebuilt, treatment with metronidazole or clindamycin does not prevent recurrence of $\mathrm{BV}$ infection. ${ }^{[24-25]}$

Therefore, one could envisage possible clinical advantages for the use of biotherapeutic agents (prebiotics / probiotics / symbiotics) together with the $\mathrm{CDC}$ standard regimen for BV eradication and restoration of healthy vaginal microenvironment. Considering BV as a predisposing factor for transmission of other STIs and BV as a common worldwide vaginal infection in women of childbearing age, it becomes clear that the association between $\mathrm{BV}$ and other STIs, i.e. controlling and normalizing the vaginal microbiome in all women of childbearing age, is an relatively inexpensive and appropriate strategy for fighting STI transmission and infection. ${ }^{[24-25]}$

A controlled clinical trial including over 800 females with BV who were treated with the usual regimen (Metronidazole) followed with long-term symbiotic vaginal therapy found a dramatic decrease in BV recurrence. Application of this topical symbiosis for at least six months resulting restoration of the normal vaginal microflora (eubiosis). These data support the long-term use of probiotics following the use of standard therapy regimen recommended by the CDC in restoring vaginal eubiosis and in controlling the transmission of sexually transmitted infections. This inexpensive and simple therapy can be a steppingstone for future preventive and curative efforts for sexually transmitted infections. ${ }^{[24-25]}$ 


\section{CONCLUSION}

Sexually transmitted infection (STI) is one of the global health problems not yet completely managed, with increasing prevalence and incidence rates from year to year. Many studies and literature have revealed a significant relationship between the microbiome and various STIs. Microbiome-based therapeutic approach holds great promise for future STI management.

\section{REFERENCES}

1. World Health Organization. Sexually Transmitted Infections (STIs) [Internet]. World Health Organization. 2019 [Cited Jan 29 ${ }^{\text {th }} 2020$ ]. Available from: https://www.who.int/news-room/fact-sheets/detail/sexually-transmitted-infections-(stis)

2. Brotman RM. Vaginal Microbiome and Sexually Transmitted Infections: An Epidemiologic Perspective. J Clin Invest. 2011; 121(12): 4610-7.

3. Cone RA. Vaginal Microbiota and Sexually Transmitted Infections that May Influence Transmission of Cell-Associated HIV. J Infect Dis. 2014; $210(3)$ : S616-21.

4. Mestrovic T. Vaginal Microbiome and Sexually Transmitted Infections. Medical and Life Sciences [Internet]. NEWS Medical Life Sciences. 2016 [Cited Jan 29 2020 ]. Available from: https://www.news-medical.net/health/Vaginal-Microbiome-and-SexuallyTransmitted-Infections.aspx

5. Berg G, Rybakova D, Schloter M. Microbiome Definiton Re-Visited: Old Concepts and New Challenges. Microbiome. 2020; 8(103): $1-22$.

6. World Health Organization. Global Health Strategy on sexually Transmitted Infections 2016 - 2021. Switszerland: WHO. 2016 p 164.

7. Ho K. Sexually Transmitted Infections in HIV/AIDS [Internet]. 2016 [Cited on Jan 29 ${ }^{\text {th }}$ 2020]. Available from: https://www.aids.gov.hk/pdf/g190htm/39.htm

8. Mandar R. Microbiome of Male Genital Tract: Impact on the Health of Man and His Partnes. Pharmacological Research. 2013; 69: 3241.

9. Lewis FMT, Bernstein KT, Aral SO. Vaginal Microbiome and Its Relationship to Behavious, Sexual Health, and Sexually transmitted infections. Obstet Gynecol. 2017; 129(4): 643-54.

10. Ma Z, Li L. Quantifying the human vaginal community state types (CSTs) with the species specificity index. Peer J. 2017 ; 5: e3366.

11. Torcia MG. Interplay among Vaginal Microbiome, Immune Response, and Sexually Transmitted Viral Infection. Int J Mol Sci. 2019; 20(2): 266.

12. Park YJ, Lee HK. The Role of Skin and Orogenital Microbiota in Protective Immunity and Chronic Immune-Mediated Inflammatory Disease. Front Immunol. 2018; 8: 1955.

13. Wijgert JHHMVD. The Vaginal Microbiome and Sexually Transmitted Infections are Interlinked: Consequences for Treatment and Prevention. PLoS Med. 2017; 14(12): e1002478.

14. Onderdonk AB, Delaney ML, Fichorova RN. The Human Microbiome during Bacterial Vaginosis. Clin Microb Rev. 2016; 29(2): 22338.

15. Widiatma RR, Listiawan MY, Murtastutik D, et al. The Efficacy of Lactobacillus plantarum as Adjunctive Therapy of Bacterial Vaginosis: A Double-blind, Randomized, Placebo-controlled Trial. BIKKK. 2020; 32(3): 214-20.

16. Molenaar MC, Singer M, Ouburg S. The Two-Sided Role of Vaginal Microbiome in Chlamydia trachomatis dan Mycoplasma genitalium Pathogenesis. Journal of Reproductive Immunology. 2018; 130: 11-7.

17. Foschi C, Salvo M, Cevenini R, Parolin C, Vitali B, Marangoni A. Vaginal Lactobacillus reduce Neiserria gonorrhoeae Viability through Multiple Strategies: An in Vitro Study. Front Cell Infect Microbiol. 2017; 7-501: 1-10.

18. Petrova MI, Broek MVD, Balzarini J, Vanderleyden J, Lebeer S. Vaginal microbiota and its role in HIV transmission and infection. FEMS Microbiol Rev. 2013; 37(5): 762-92.

19. Karim SSA, Baxter C, Passmore JS, McKinnon LR, Williams BL. The Genital Tract and Rectal Microbiomes: Their Role in HIV Susceptibility and Prevention in Women. J Int AIDS Soc. 2019; 22(5): e25300.

20. Eastment MC, McClelland RS. Vaginal Microbiota and Susceptibility to HIV. AIDS. 2018; 32(6): 687-98.

21. Nardis C, Mosca L, Mastromarino P. Vaginal microbiota and viral sexually transmitted infections. Ann Ig. 2013; 25(5): 443-56.

22. Cheng L, Norenhag J, Hu YOO, Brusselaers N, Fransson E, et al. Vaginal microbiota and human papillomavirus infection among young Swedish women. NPJ Biofilms and Microbiomes. 2020; 6(1): 39-48.

23. Lin D, Kouzy R, Jauede JA, Noticewala SS, Medrano AYD, et al. Microbiome factors in HPV-driven carcinogenesis and cancers. PLoS Pathog. 2020; 16(6): e1008524.

24. Bertini M, Baldacci L. Is Time for Thinking to Vaginal Microbiome to Prevent Sexually transmitted infection?. Int J Fam Commun Med. 2018; 2(3): 24-6.

25. Bolton M, Straten A, Craig C. Probiotics: Potential to Prevent HIV and Sexually Transmitted Infections in Women. Sex Transm Dis. 2008; 35(3): 214-25. 